\title{
Equivalent block transmissivity in an irregular 2D polygonal grid for one-phase flow: a sensitivity analysis
}

\author{
Marco De Lucia ${ }^{1,2}$, Chantal de Fouquet $^{1} *$, Vincent Lagneau ${ }^{1}$
}

October 7, 2008

1 École des Mines de Paris

${ }^{2}$ Université de Bologne

* corresponding author: Centre de Géosciences, Mines ParisTech - Ecole des Mines de Paris, 35 rue Saint Honoré, 77305 Fontainebleau Cedex, France, tel: (33)1 646947 61, fax: +33 164694761 , chantal.de_fouquet@ensmp.fr

\begin{abstract}
Upscaling is needed to transform the representation of non additive space-dependent variables, such as permeability, from the fine grid of geostatistical simulations (to simulate small scale spatial variability) to the coarser, generally irregular grids for hydrodynamic transport codes. A new renormalisation method is proposed, based on the geometric properties of a Voronoï grid. It is compared to other classic methods by a sensitivity analysis (grid, range and sill of the variogram, random realisation of a simulation); the criterion is the flux of a tracer at the outlet. The effect of the upscaling technique on the results appears to be of second order compared to the spatial discretisation, the choice of variogram, and the realisation.

Keywords: Keywords: equivalent permeability, upscaling, finite volumes, voronoï mesh, spatial variability, heterogeneous medium, flow
\end{abstract}

\section{Résumé}

Le changement d'échelle pour la perméabilité est incontournable, entre une simulation géostatistique sur grille fine (reproduisant la variabilité spatiale à petite distance) et le maillage plus grossier et généralement irrégulier des codes de transport. Une nouvelle méthode de renormalisation, exploitant les propriétés géométriques du maillage de Voronoï, est comparée à deux méthodes classiques, par une étude de sensibilité (maillage, portée et palier du variogramme, tirage ou « réalisation » d'une simulation) suivant un critère de flux d'un traceur en sortie de perméamètre. La technique de changement d'échelle apparaît du second ordre par rapport à la discrétisation spatiale, au variogramme et à la réalisation.

Mots-clés: permeability équivalente, changement d'échelle, volumes finis, maillage de Voronoï, variabilité spatiale, milieu hétérogène, écoulement

\section{Introduction}

Upscaling is a required step to adapt a fine grid geostatistical simulation (to reproduce spatial variability at different scale) to equivalent parameters on (usually irregular) grids used by transport codes. A new renormalisation method is proposed, based on the properties of a Voronoï grid used in the code HYTEC, to compute the inter-block permeability; this method does not require the knowledge of the local flow direction. Our computation of the scalar block permeability is compared to two other classical renormalisation methods, based on a parametric sensitivity analysis to the spatial variability, in dimension 2. The chosen observable is the cumulative flux of a non-reactive tracer through the outlet of the domain. 


\section{Bibliographical elements}

Since Renard and de Marsily synthesis [19], developments on upscaling methods are still carried on, in various domains: multiphase flow [6], fractured media [10, 11, 21], reactive transport [14], etc. Two general classes of methodologies can be highlighted: on the one hand the cell aggregation, which aims at adapting the grid to the medium's properties and the flow boundary conditions [1,3,12]; on the other hand, methods which aim at attributing equivalent values (or at least an interval) for properties on a fixed geometry. The second seems better adapted for numerical models with a high spatial variability [7], particularly when permeability or transmissivity are obtained by geostatistical simulations based on the anamorphosed gaussian model. Furthermore, for reactive transport applications, the possible evolution of the pore structure, due to precipitation or dissolution reactions, is still a serious limitation to the use of adaptive grids.

\subsection{Block permeability: exact results or inequalities}

The equivalent permeability of a block is precisely known in a few particular cases [16]. According to the fundamental inequality, a block permeability is bounded by the harmonic- and the arithmetic-mean of its elements. When the permeability is simulated on a fine grid, the interval can become very large, if the blocks are constituted of numerous fine cell elements, or if the dispersion variance of the permeability of the cells inside the block is high. Several authors suggested tighter bounds, for particular media or boundary conditions [18]. For instance, for relatively general conditions (linear pressure or constant flux on the boundary), Pouya [17] shows the rise of two particular tensors, linked by an inequality: equivalent permeabilities in the direction of the mean gradient and the mean flux respectively. He then proposes to use these tensors as upper and lower bounds for the results, according to the problem to solve. While bounding the result is more rigourous, "realistic" values are sometimes sufficient, particularly when the computation times are large as in reactive transport.

\subsection{Empirical formulas}

For lack of a general rule for permeability composition, several empirical formulas have been studied, notably renormalisation methods: iterative treatments to remove small scale fluctuations [8]. In the following, we retain two expressions of block permeability.

The first one is based on Matheron's formula [16], a composition of the arithmetic mean $\mu_{a}$ and the harmonic mean $\mu_{h}$, as a function of the dimension $D$ of the space:

$$
K_{M}=\left(\mu_{a}\right)^{\alpha}\left(\mu_{h}\right)^{1-\alpha}
$$

where $\alpha=\frac{D-1}{D}$. In dimension 2 ,

$$
K_{M}=\sqrt{\mu_{a} \cdot \mu_{h}}
$$

so that the equivalent transmissivity is the geometric mean of the harmonic and the arithmetic means of the transmissivities of the cells inside the block. This formula has since been generalised, e.g. [2, 4].

The second method is based on the simplified renormalisation by Renard [18, 20]: it consists in an iterative composition of the values of the elementary cells contained by the block, alternating arithmetic and harmonic means. This method yields two bounds, following each axis of the grid: the lower (resp. upper) bound $c_{\min }\left(r e s p . c_{\max }\right)$ is obtained when the first iteration uses a harmonic (resp. arithmetic) mean. These bounds are then composed by power-average, taking into account the medium anisotropy if needed. Let $u$ the assumed direction of the tensor, taken parallel to one of the axes for simplicity; let $c_{\min }^{u}\left(\right.$ resp. $\left.c_{\max }^{u}\right)$ the associated lower (resp. upper) bound; let $K^{u}$ the diagonal component of the permeability tensor on the elementary cell in this direction. Then, for a $2 \mathrm{D}$ isotropic medium, the simplified renormalisation is the geometric mean of the two bounds given by the alternating means:

$$
K_{S R}^{u}=\sqrt{c_{\max }^{u} \cdot c_{\min }^{u}}
$$

This relationship is similar to Matheron's formula, but applies with tighter bounds. For an anisotropic medium, the upscaling transforms a scalar permeability into a tensorial permeability. If the flow solver accepts scalars values only, the block permeability can be computed by assuming the direction of the head 
gradient known. In 2D, for a diagonal permeability tensor in the directions $(O x, O y)$, the permeability along the flow direction $\vec{s}=(\cos \theta, \sin \theta)$ is:

$$
K_{R S}=\cos \theta \cdot K_{S R}^{X}+\sin \theta \cdot K_{S R}^{Y}
$$

When the local direction of the flow $\vec{s}$ is not known, the consistency of the equivalent permeability obtained by this method decreases when the effective direction of $\vec{s}$ diverge from its supposed direction.

\section{Irregular grid renormalisation}

In his Ph.D thesis, Renard extends the simplified renormalisation to irregular blocks, by weighting the successive means depending on the blocks geometry ([18], p. 92-93). In the following, we investigate the transmissivity composition for a 2D medium, discretised by an irregular grid. While we work in the plane, we still use the term "permeability": this is consistent, e.g., with a flow in a constant depth aquifer. The flow code is briefly introduced, then a new upscaling method is proposed, based on the specific properties of the spatial discretisation scheme.

\subsection{The Hytec code: finite volumes on a Voronoï polygons grid}

HYTEC [14] is a coupled hydrodynamic and geochemistry code. It is based on the resolution of the macroscopic equations of flow and advective/dispersive transport on a finite volumes scheme (or integrated finite differences), using a spatial discretisation by irregular Voronoï polygons. The convex polygons are built, starting from an arbitrary set of points, by intersection of the perpendicular bisector of each pair of nodes. In a finite volumes formulation, all parameters are considered uniform inside each polygon.

For a one-phase stationary flow, HYTEC solves the diffusivity equation and the associated Darcy's equation [15]:

$$
\left\{\begin{aligned}
0 & =\operatorname{div}(K \cdot \overrightarrow{\operatorname{grad}} h) \\
\vec{U} & =-K \cdot \overrightarrow{\operatorname{grad}} h
\end{aligned}\right.
$$

where $h$ is the head (in $\mathrm{m}$ ), $\vec{U}$ is the Darcy velocity vector (in $\mathrm{m} / \mathrm{s}$ ), and $K$ the permeability (in $\mathrm{m} / \mathrm{s}$ ). The equation is resolved with respect to the unknown $h$; then the normal component of $\vec{U}$ is computed, on each cell boundary, via Darcy's equation. Once determined the flow velocity field, the transport equation is written:

$$
\frac{\partial c}{\partial t}=\operatorname{div}\left(\left(D_{e}+\alpha\|\vec{U}\|\right) \cdot \overrightarrow{\operatorname{grad}} c-c \cdot \vec{U}\right)
$$

where $c$ is the tracer concentration, $D_{e}$ is the effective diffusion coefficient (in $\mathrm{m}^{2} / \mathrm{s}$ ) and $\alpha$ the dispersivity (in $\mathrm{m}$ ).

HYTEC proposes a choice of algorithms for the resolution of the transport equation. The time and space interpolations are one-step, but the centring can be configured by the user. We chose for this study a time-centred pattern (Crank-Nicholson scheme), which has little effect on the result apart from the maximum admissible time step.

As to the space discretisation, HYTEC proposes both an upwards and a centred scheme. The first is unconditionally stable, but creates a numerical dispersion term (mathematically equivalent to a physical dispersivity $\alpha$ ), locally of the order of a half-cell diameter. The second scheme is space-centred: it does not create numerical dispersion, but its stability is conditional to the local value of $\alpha$. The first scheme is generally preferred for coupled reactive transport problems: indeed, the geochemistry usually limits the effects of dispersion by a chemical sharpening of the fronts. However, the numerical dispersion is proportional to the (local) size of the cells, so that an accurate comparison between several grid computations is difficult. The effects of the space centring are discussed further in the results section 5 .

\subsection{Simplified renormalisation for a polygonal cell}

The simplified renormalisation algorithm can be readily adapted to an irregular mesh, by weighting the means at each iteration according to the actual number of cells inside the polygon. This is indeed a local algorithm. 

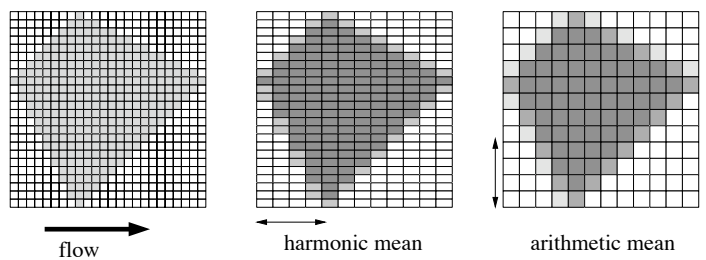

Figure 1: Simplified renormalisation on a polygonal cell. When the flow direction is known a priori, the renormalisation is performed on the circum-rectangle, with weighting according to the relative surface of the blocks inside the polygon.

In the following, a "cell" will refer to the fine regular mesh of the geostatistical simulation. The simplified renormalisation algorithm on an irregular mesh is then as follow (see figure 1):

1. each polygonal mesh is discretised into cells, following the circum-rectangle of the polygon;

2. a weight 1 is attributed to each cell if the centre of the cell is inside the polygon, 0 otherwise;

3. the permeabilities of the rectangle are iteratively composed, weighted by the sum of the cells used at the iteration; therefore, if the centre of mass of a cell falls out of the polygon, the permeability of the cell is not taken into account.

The last step of the algorithm is repeated until a unique value is obtained for each polygon, for the considered mean.

\subsection{Normal component renormalisation}

Using the properties of a Voronoï mesh, we can introduce an empirical upscaling, without hypothesis on the flow direction and on the principal directions of the permeability tensor. Indeed, the mass balance equation is computed using only the normal component of the flux between adjacent polygons.

For each polygon, an equivalent permeability is computed by splitting the polygon as a sum of triangles: their bases are two adjacent vertices of the polygon and their summit the centre of the polygon (figure 2, right). By construction of the Voronoï mesh, the triangles with common base from two adjacent polygons are identical, in particular their surface areas are the same. These triangles are gathered by pairs to form "kite" figures. The inter-block permeability used by the finite volume scheme is precisely the block permeability of these quadrilaterals, following a similar reasoning to [9]. In the mass balance, only the normal component of the flow through the shared side of two adjacent polygons is used; for this normal flow, the equivalent permeability can be computed, e.g., by simplified renormalisation.

Due to historical choices, the code HYTEC uses only scalar components: indeed, in coupled reactive transport phenomena, the geochemistry often limits the effects on the transport of a possible anisotropy of the medium. Assuming isotropic conditions, the diagonal terms of the permeability tensor obtained by this method are combined following the direction of the normal flow, which is determined by the mesh geometry (by construction of the Voronoï mesh). This inter-block permeability is used directly in the HYTEC code: it corresponds to the mid-nodal permeability of finite differences. It is worth mentioning that the discretisation obtained by this method is finer than the finite volumes partition of space for which permeability would be uniform inside each polygon. To preserve the consistency with the finite volume formalism, a intra-block upscaling of the permeability can also be computed: each polygon can be divided in as many triangles as it has vertices (two adjacent vertices form the basis of the triangle, the centre of the polygon forms its summit); on these triangles, the simplified renormalisation yields a value of the equivalent permeability for a flow normal to the opposite side. The equivalent permeability are then composed to get the equivalent permeability of the polygon; an arithmetic composition has been chosen, weighted by the relative surface of each elementary triangle of the polygon.

For anisotropical cases, the reasoning is not so easy. Beyond the necessity to take the permeability tensor into account, it is not possible anymore to use the simplification based on the normal component of the flow at each polygon interface comment, so that the renormalisation scheme has to be heavily modified. 


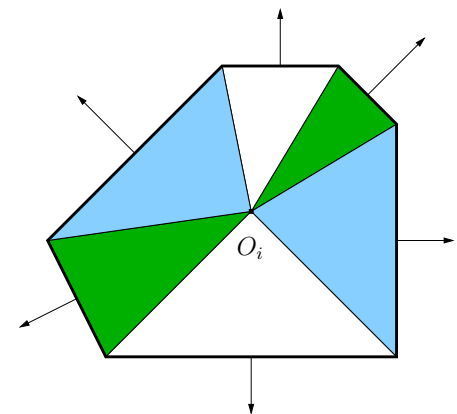

(a) A polygonal cell is split in a sum of triangles

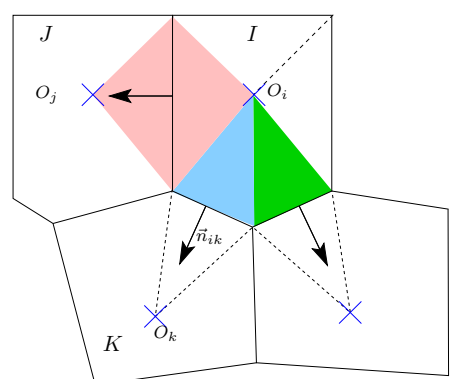

(b) Inter-block assessment

Figure 2: Renormalisation along the normal component. The geometry of the grid gives the local direction of the normal flow. For the intra-block folrmulation, the permeability is computed on each sub-triangle, with a final recomposition to obtain the equivalent permeability of the polygon. For the inter-block formulation, the equivalent permeability on each quadrangle (pair of adjacent triangles) is provided as is to the hydrodynamic model.

In the following, all three modes of composition are tested: Matheron's mean, simplified renormalisation, renormalisation along the normal component of the flow. Each yields two values: an intra-block permeability (uniform inside each polygon), and an inter-block permeability (on the quadrilateral between the centre and adjacent side of each pair of adjacent polygons). For the simplified renormalisation, the (intra- or inter-block) scalar permeability is computed assessing a local direction of the flow parallel to the macroscopic head gradient, determined by the boundary conditions.

\section{Design of the numerical experiments}

The sensitivity study aims at discriminating between the influence of the spatial variability of the medium (represented by the geostatistical simulation on the fine grid) and the representation of this variability, which depends on the spatial discretisation for the finite volumes and the upscaling scheme. To this end, numerical experiments are carried out on geostatistical simulations of the permeability only; inter- or intra-block equivalent values are computed using all three upscaling methods, for several types of HYTEC grids.

The domain of study is a permeameter: it consists in a rectangle, with a uniform inflow along a boundary, a uniform constant head condition along the opposite boundary, while the other two boundaries are impermeable (figure 3). The permeameter has been extended with two lines of cells just outside the control lines, for better control of the boundary conditions independently of the polygonal grid inside the permeameter. At initial time, a perfect tracer $A$ is at constant concentration 1 inside the permeameter; the incoming flow flushes the permeameter with a tracer $B$ at concentration 1 (and no tracer $A$ ).

In the bulk of the permeameter, the permeability $K$ is assumed lognormally distributed, obtained from a (multi) gaussian random function $Y$ :

$$
K=M e^{\sigma \cdot Y-\frac{\sigma^{2}}{2}}
$$

where $M$ is the arithmetic mean, and $\sigma^{2}$ the logarithmic variance of the permeability. In this model, the spatial variability is characterised by the variogram $\gamma$ of the random function $Y$. Once fixed the variogram model type (spherical, exponential, ...), the set of parameters which fully describe the spatial model is limited to the range $a$ (correlation length) and the standard deviation $\sigma_{\log K}$ of the logarithm of the permeability. Due to the choice of boundary conditions, the flow is independent of the mean $M$ of the permeability. We chose $M=10^{-3} \mathrm{~m} / \mathrm{s}$.

The results of the study are all presented adimensionally; e.g. the length of the field is fixed to $L=100 \mathrm{~m}$ : all the other parameters of the same dimension (range of the variogram, dispersivity, ...) are given relative to the unit $L$. For the hydrodynamics resolution, the time is expressed adimensionally, 


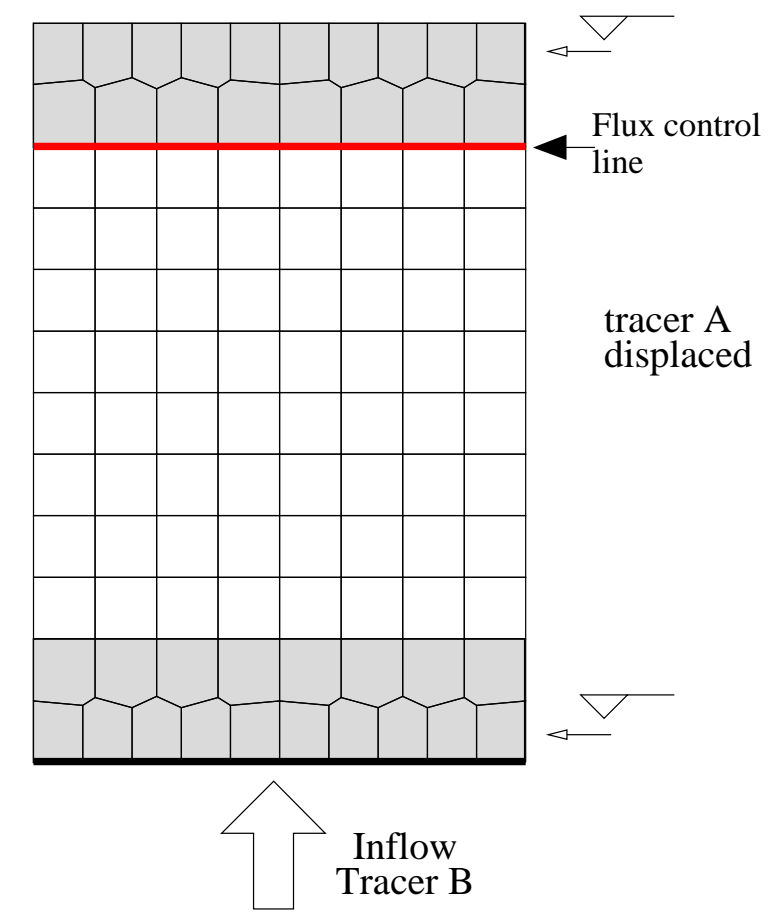

Figure 3: Boundary conditions for the numerical experiments.

as a number of pore volumes injected (the total pore volume is equal to the surface of the permeameter in 2D multiplied by its mean porosity); we refer to this adimensional time also as "water renewal cycles".

The observable chosen to describe the system is the cumulative flux of tracer through a control line at the outlet of the permeameter: tracer $A$, initially at concentration 1 inside the permeameter, is flushed out by the injection of fresh ( $A$-free) water at the inlet. Throughout the study, we will show the cumulative flux of $A$ through the outlet after an adimensional time 0.8 (i.e. after $80 \%$ of the pore volume has been renewed). This particular time is particularly interesting: later on, most of the initial tracer has been flushed, so that the cumulative fluxes are close to the initial total amount of $A$; for shorter amounts of time, the effects of the spatial variability are still limited to the neighbourhood of the inlet, and have low effects on the fluxes at the outlet.

Two series of numerical experiments were carried out. The first one is based on a $64 \times 64$ geostatistical simulation, which allows for a HYTEC reference simulation, without preliminary upscaling. The upscaling techniques are then applied to build coarser HYTEC grids: $16 \times 16$ and $8 \times 8$ rectangular grids. For both size, three classes of HYTEC grids are investigated: regular rectangular, diamonds (indeed $45^{\circ}$-tilted rectangles), and rectangular with diamond inclusion (figure 4); so that six coarse grids are investigated. For the second series of numerical experiments, $500 \times 500$ reference simulations have been attempted. However, the implicit geochemistry in HYTEC (even for non-reactive tracer) lead to a degradation of the precision of the results for such fine grids; in our specific case, where precise comparisons of fluxes through a boundary are performed, the quality of the results were not sufficient to provide a definite reference. This point should be improved in further versions of HYTEC.

For the first series of experiments, the $64 \times 64$ geostatistical simulations were performed by the discrete spectral method: the method is very CPU-effective, and offers the possibility to vary the range $a$ at "constant random draw", i.e. using the same realisation of random numbers. We can thus investigate the effect of the range without interference from the random variability between two draws [5]. For the second series of experiments, we chose the turning bands method; the code has not been adapted for this specific purpose, so that the $500 \times 500$ grid simulations for different ranges correspond to different random draws.

Three values were tested for the range of the spherical variogram of the logarithm of the permeability (equation 3): $a=10,30$ and $50 \%$ of the length $L$ of the permeameter. Five values were chosen for the 


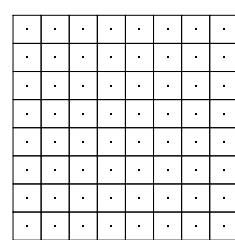

(a) Regular family (b) Diamondshape family

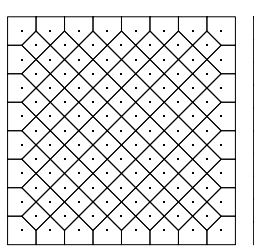

(c) Inclusion family

Figure 4: Grids for the second series of numerical experiments.

logarithmic standard deviation $\sigma_{\log K}$ :

\begin{tabular}{lccccc}
\hline Variance $\left[m^{2} / s^{2}\right]$ & $10^{-4}$ & $5 \cdot 10^{-5}$ & $10^{-5}$ & $5 \cdot 10^{-6}$ & $10^{-6}$ \\
$\sigma_{\log K}$ & 2.15 & 1.98 & 1.55 & 1.34 & 0.83 \\
\hline
\end{tabular}

The other parameters were chosen as follow:

- high dispersivity: $\alpha=0.1 \times L$;

- uniform porosity: $\omega=0.3$;

- effective diffusion coefficient: $D_{e}=10^{-10} \mathrm{~m}^{2} / \mathrm{s}$.

It is important to note the need of a careful control of the computation conditions on the precision of the result, for the chosen observable, particularly, the time step. HyTEC determines an optimal time step, following several criteria (e.g. speed of convergence for the coupling); the maximum time step is bounded by the Courant-Friedrich-Levy stability criterion; however, a precise comparison between computations at different time discretisation revealed that the criterion was not stringent enough. We finally made all simulations with the same time step, based on the smaller time step obtained for the finer grid.

\section{Experiments results}

The transport computations were performed in an advective/dispersive framework, with a dispersion around $10^{3}$ times larger than diffusion. The dispersivity coefficient is quite high, around 0.1 times the length of the permeameter.

\subsection{Simulations without numerical dispersion}

The transport simulation using the centred scheme, without numerical dispersion, show that the cumulative fluxes on a homogeneous medium are independent of the grid. In figure 5 , the cumulative fluxes curves are strictly identical for the 64,16 and 8 grids.

On the contrary, the upward scheme, with added numerical dispersion, produces a delay on the arrival of the tracer; the delay increases with the size of the polygons. The cumulative flux curves converge towards the theoretic limit (a proof that the code does not loose mass), but the time needed to converge towards the limit increases with coarser grids: indeed, for coarse grids, the numerical dispersion is larger, which clearly creates longer dispersion tails, and delays the complete flushing of the tracer.

In the following, results are given using the numerically dispersive scheme.

\subsection{Upscaling between rectangular cells $64 \times 64,16 \times 16$, and $8 \times 8$}

The parametric study is performed systematically using the same initial random realisation, using the several values for the geostatistical parameters: 5 values for $\sigma, 3$ for the range, 3 renormalisation methods, intra- or inter-block variation, fine initial grid and the two other grids. 


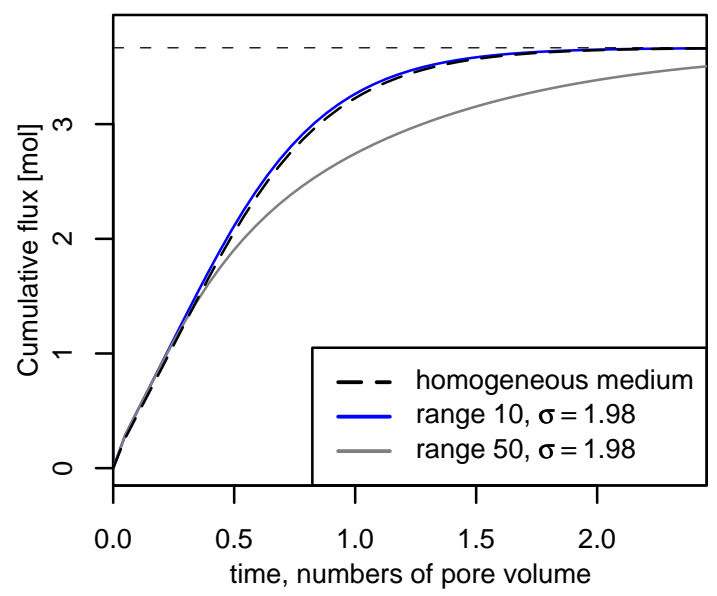

Figure 5: Cumulative flux for the $64 \times 64$ reference grid; homogeneous permeability, range $0.1 \mathrm{~L}$ and $0.5 \mathrm{~L}$ and large standard deviation.

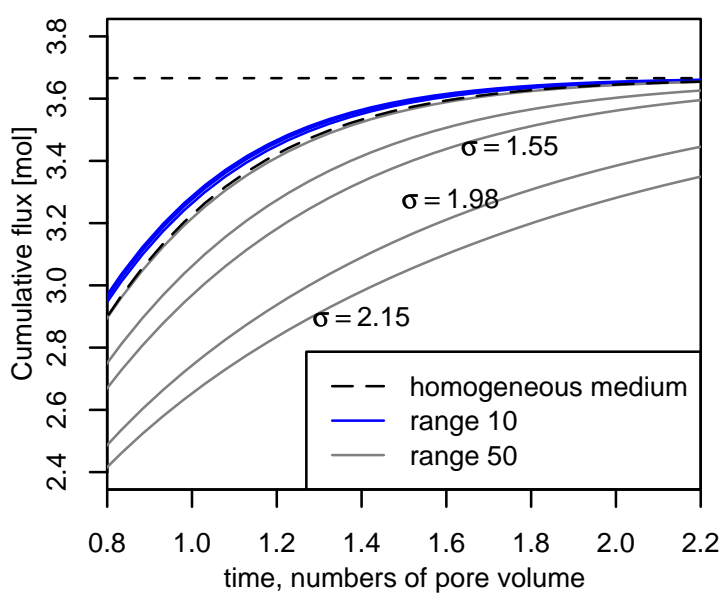

Figure 6: Cumulative flux for the $64 \times 64$ reference grid, compared to the homogeneous case. For the larger range, the logarithmic variance has a large impact; on the contrary, for the smaller range (in blue), the differences are very small.

Figure 6 shows the cumulative fluxes obtained on the reference simulation $(64 \times 64$ grid $)$, for media with range $0.1 L$ and $0.5 \mathrm{~L}$. For $a=0.1 \mathrm{~L}$, the curve are superimposed for all variance values, and close to the uniform permeability reference. For $a=0.5 L$, the variance plays a major role: the cumulative fluxes diverge from the uniform permeability reference. A possible explanation is that longer range geostatistical simulations create spatially well-structured fluctuation in the flow velocity field; increasing variances accentuate the discrepancy between low and high permeability zones, so that the effect of preferential pathways increases.

The spatial variability of permeability systematically introduces a delay on the cumulative fluxes curves, compared to the homogeneous reference or small range or small variance simulations. On the short term, preferential pathways allows for a faster circulation of the tracer; however, on the longer term, slow circulation areas delay the complete flush of the tracer. The spatial variability has thus a dual effect on the system behaviour: the breakthrough of the tracer $B$ is faster, but the complete leaching of the initial tracer $A$ takes longer.

Let $Q_{\text {ref }}(t)$ be the cumulative flux in the reference simulation, on the initial $64 \times 64$ grid, and $Q_{\text {ups }}(t)$ the results after upscaling. Figures 7 through 8 show the effect of the upscaling, as the difference 


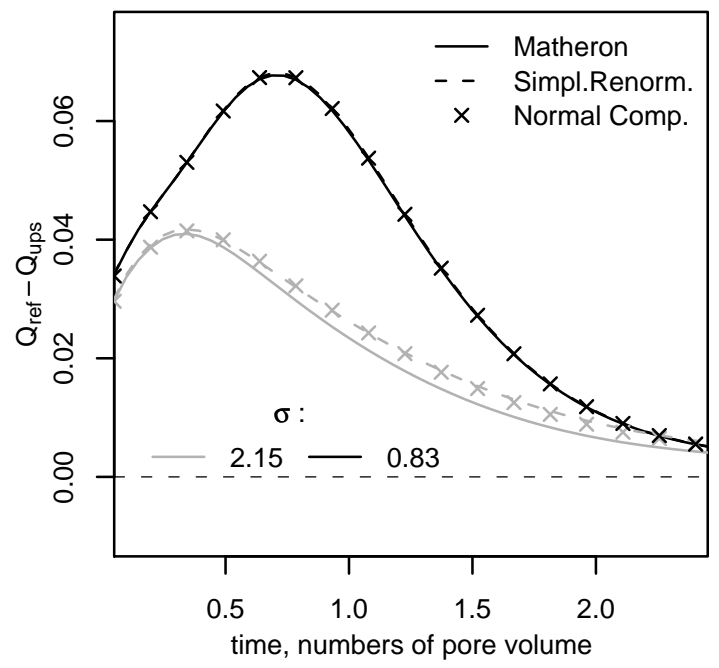

Figure 7: Difference of the cumulative flux relative to the reference simulation; $16 \times 16$ grid, inter-block formulation, range $0.3 \mathrm{~L}$.

$Q_{\text {ups }}(t)-Q_{\text {ref }}(t)$. Indeed, in this case, the ratio $Q_{u p s} / Q_{\text {ref }}$ tends to decrease the difference between simulations results, so that it is not a good discriminating observable.

The variance of the permeability discriminates between the curves. Unexpectedly, the maximum difference compared to the reference is obtained for small values of the variance. This can be explained by the transformations due to the upscaling, which modify the preferential pathways for a small variance. On the contrary, for larger values of $\sigma$, the preferential pathways are more pronounced, and resist to all the upscaling techniques. The differences between upscaling methods can be seen on the $16 \times 16$ grid (figures 7 and 9), and even more on the $8 \times 8$ grid (figures 10 and 8 , with different scales than for the $16 \times 16$ grid figures).

Logically, the effect of the range on the upscaling is more related to its ratio to the mean size of the blocks. For the same range, the influence of the upscaling (of the hydrodynamic grid) increases with the block sizes (figures 7, 10 on the one hand and 9, 8 on the other hand). For a fixed hydrodynamic grid, the differences between the three upscaling methods increase with the range (figures 7, 9 on the one hand and 10, 8 on the other hand), and is greater when the variance increases.

It is not straightforward to set a hierarchy between the upscaling methods. For the inter-block method, the results of the normal component renormalisation are a little closer to the reference, compared to the simplified renormalisation. The Matheron's mean is systematically less precise for the $8 \times 8$ grid, but better for the $16 \times 16$ grid.

\subsection{Experiments on complex grids}

A second series of numerical experiments were carried out, with higher relative influence of the upscaling, and with more complex spatial discretisations for the hydrodynamic resolution (figure 4). For that purpose, the geostatistical simulations were performed on a much finer grid $500 \times 500$. Two independent realisations for each range $a=0.1 L, 0.3 L$ and $0.5 \mathrm{~L}$ were drawn using the turning bands method. It was thus possible to control the influence of the random draw, but as explained in section 4, a direct comparison of simulations with different range was no longer possible. A major difference, compared to the previous section, is the absence of a reference hydrodynamic simulation, as discussed in section 4 .

The hydrodynamic simulation results for the different grids are presented via the cumulative flux of tracer $A$ through the outlet relative to the flux for the same grid with uniform permeability. In the absence of reference, this smooth the effects of the spatial discretisation.

The results are displayed in figures 11 and 12, respectively on the square $16 \times 16$ and $8 \times 8$ grid in the intra-block formulation. The different upscaling methods are represented by different line codes (dashes, 


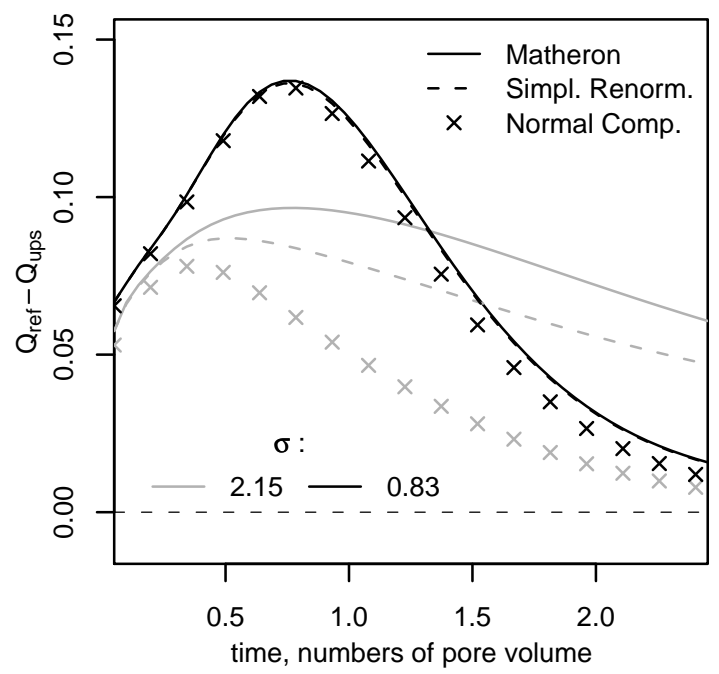

Figure 8: Difference of the cumulative flux relative to the reference simulation; $8 \times 8$ grid, inter-block formulation, range $0.5 \mathrm{~L}$.

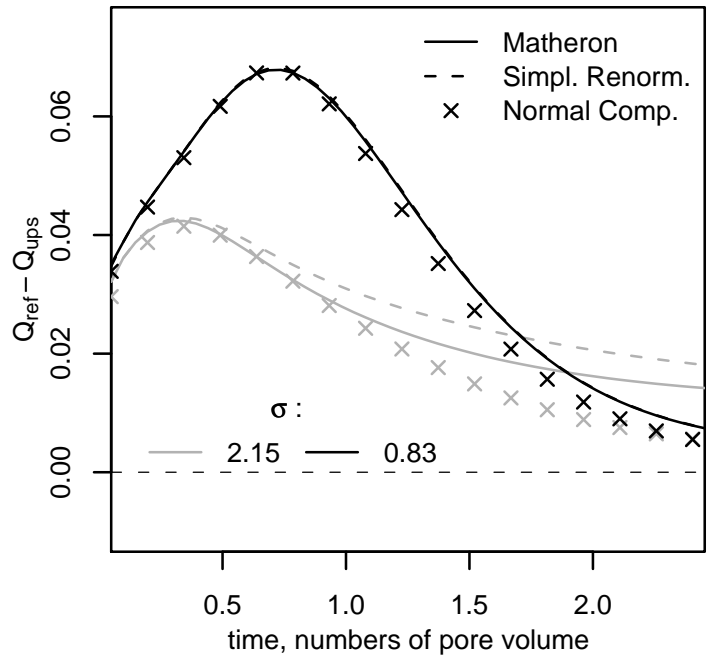

Figure 9: Difference of the cumulative flux relative to the reference simulation; $16 \times 16$ grid, inter-block formulation, range $0.5 \mathrm{~L}$. 


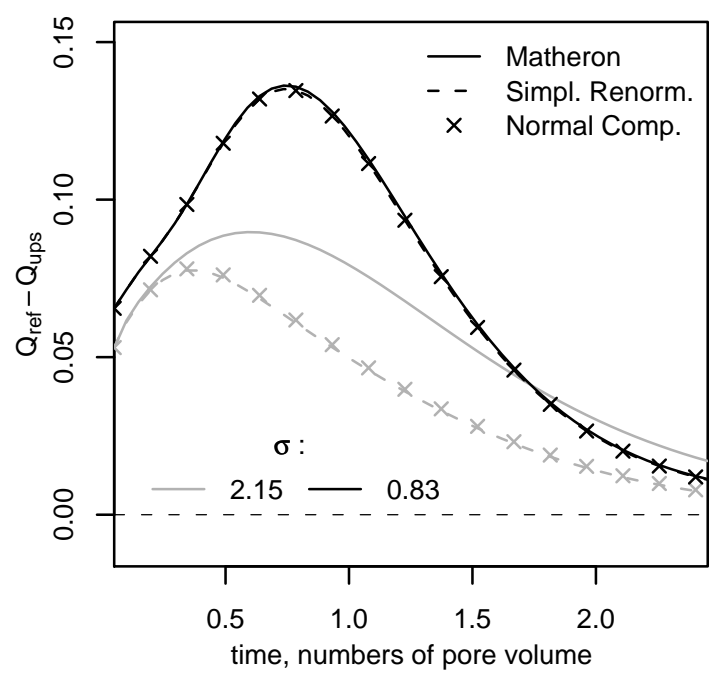

Figure 10: Difference of the cumulative flux relative to the reference simulation; $8 \times 8$ grid, inter-block formulation, range $0.3 L$.

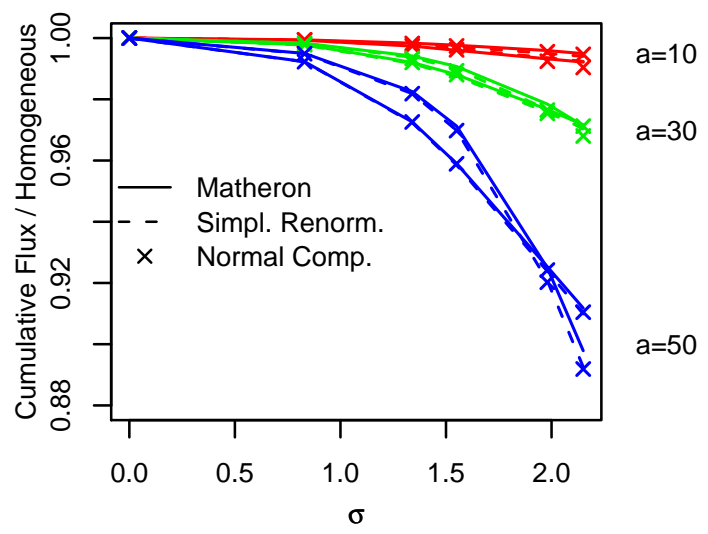

Figure 11: $16 \times 16$ rectangular grid, intra-block formulation: ratio of the cumulative flux relative to the homogeneous case as a function of $\sigma$.

dots, ...). The differences between the upscaling methods are visible only for the larger values of the range and the variance. The discrepancy also increases with larger block sizes. The results for the other types of grids (diamonds, and inclusion) give similar results.

The impact of the random draw itself is far more important than the effect of the upscaling technique, particularly for range $a=0.5 \mathrm{~L}$. In this latter case, the field is not large enough to ensure the ergodicity of the realisation: the spatial mean can thus be different from its expectation.

Owing to its limited influence (at least compared to the impact of the spatial variability itself), it is difficult to find systematic effects due to the upscaling techniques. Figures 13 and 14 show a comparison for two $16 \times 16$ and $8 \times 8$ diamond-shape grids, in intra- and inter-block respectively. The inter-block formulation seems more robust relative to the mean size of the cells. This is understandable, since the implicit underlying grid for permeability is at least twice as fine for inter- than for intra-block. At any rate, the effects of upscaling towards coarser grids is more pronounced for the intra-block formulation.

The cumulative fluxes obtained on a dense grid are generally lower than fluxes on a coarser grid (the points are below the first bisector on the scatter diagram), with the exception of the diamond-shape grids.

The figures 15 and 16 (respectively for the $16 \times 16$ and $8 \times 8$ diamond-shape grids) show a comparison 


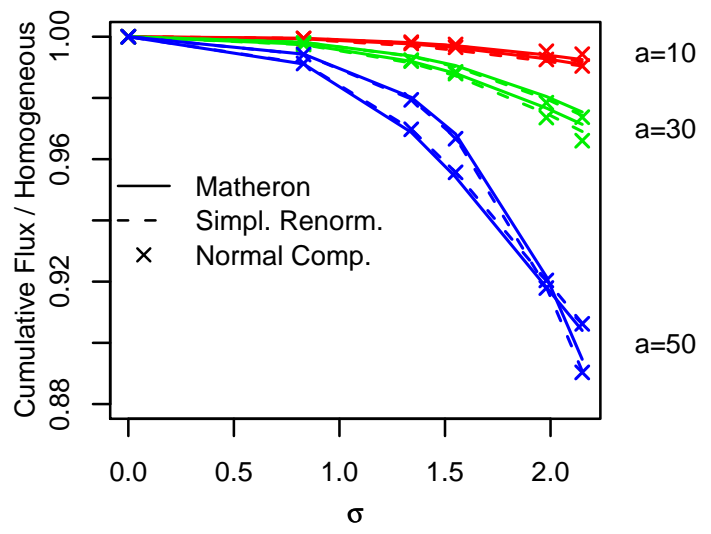

Figure 12: $8 \times 8$ rectangular grid, intra-block formulation: ratio of the cumulative flux relative to the homogeneous case as a function of $\sigma$.

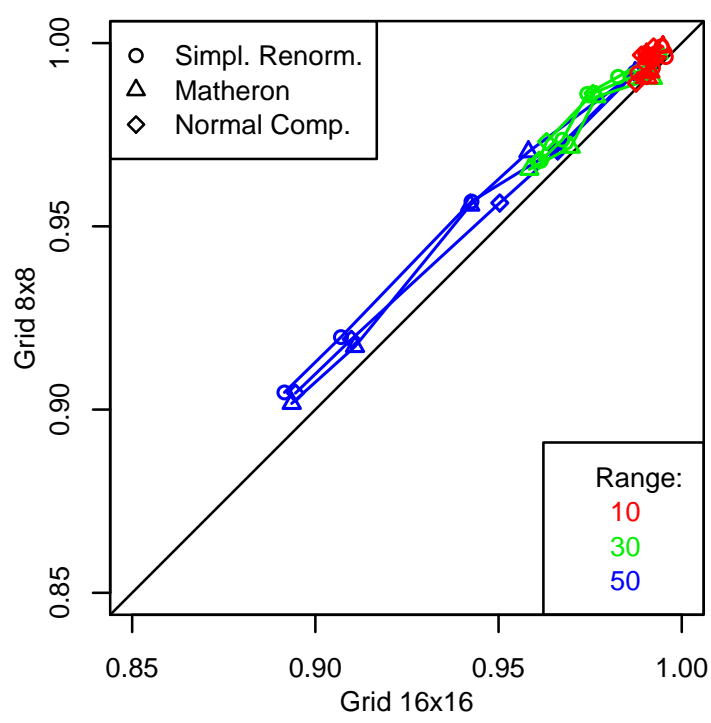

Figure 13: Scatter diagram of the cumulative flux relative to the uniform case, for several grids: diamondshape size 8 and 16, intra-block formulation, all variances together. 


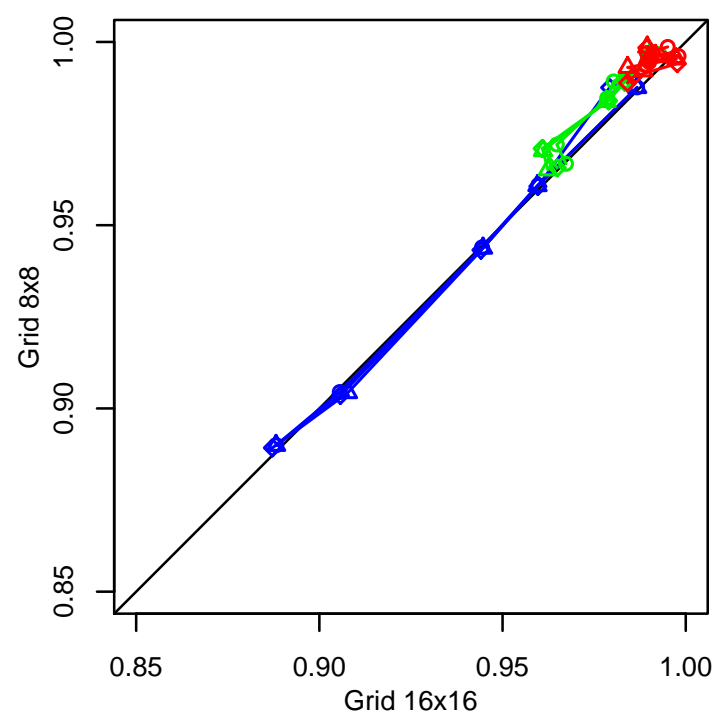

Figure 14: Scatter diagram of the cumulative flux relative to the uniform case, for several grids: diamondshape size 8 and 16, inter-block formulation, all variances together.

between the inter- and intra-block formulations. It appears that the inter-block simulations yield systematically higher cumulative fluxes than the intra-block (the points are over the first bisector on the scatter diagram).

Finally, the simulations do not show systematic differences of behaviour between the three upscaling methods.

\section{Conclusion}

Several conclusions can be drawn from the comparison of the results on different simulated media and different hydrodynamic grids. The upscaling method itself has less influence on the cumulative flux of tracer at the outlet than the actual spatial discretisation (the hydrodynamic grid), or the effective spatial variability of the medium. The discrepancy between the three upscaling techniques for the inter-block permeability is even negligible, except for the larger values of the variance of the permeability and for the larger ranges. The three techniques do not display systematic effects (e.g. under-estimation). The geometry of the hydrodynamic grid should be carefully evaluated, and should be fully considered as one of the influential parameters for the observable.

Finally, the importance of the random draw has been evidenced. Consequently, it could be worth devising a probabilistic quality criterion for the simulations; i.e., the best method should provide an unbiased estimation of the results distribution, at least in mean or better in mean and variance.

\section{Acknowledgement}

The study received a financial support by the European Community and University of Bologne. The authors thank Roberto Bruno for his support on the project. The authors are grateful for the positive reviewers, particularly Benoit Noetinger who greatly helped improve the document.

\section{References}

[1] J. E. Aarnes, V. L. Hauge, Y. Efendiev. Coarsing of three-dimensional structured and unstructured grids for subsurface flow. Adv. Water Res. 30 (2007) 2177-2193. 


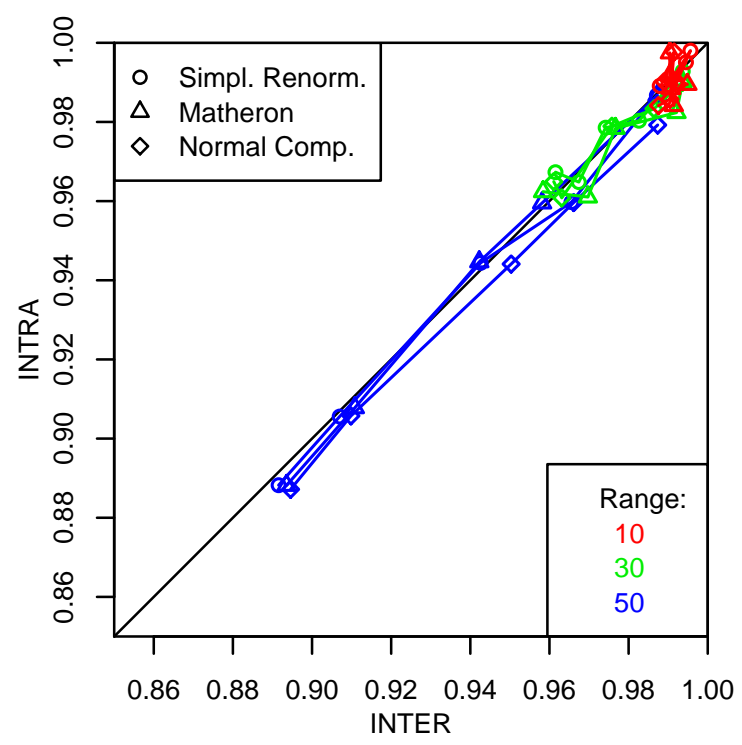

Figure 15: Scatter diagram of the cumulative flux relative to the uniform case, for inter- and intra-block formulations; $16 \times 16$ diamond-shape grid, all variances together.

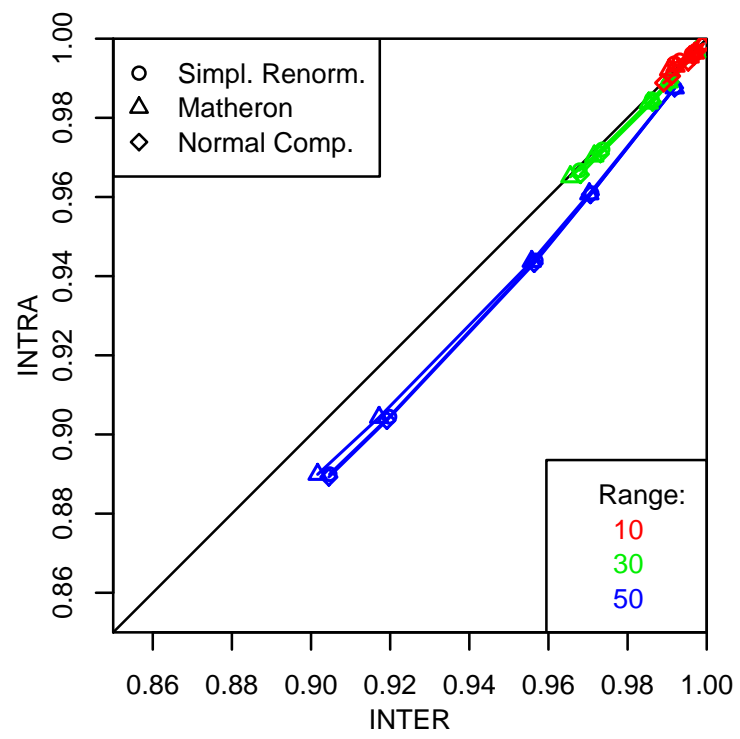

Figure 16: Scatter diagram of the cumulative flux relative to the uniform case, for inter- and intra-block formulations; $8 \times 8$ diamond-shape grid, all variances together. 
[2] R. Ababou. Random Porous Media Flow on large 3-D Grids : Numerics, Performance, and Application to Homogeneization. in IMA Vol 79. Mathematics and its applications: Environmental studies - math., computational and statistical analysis. Wheeler ed., Springer, New-York, 1996, pp1-25

[3] V. Artus, B. Noetinger. Up-scaling Two-Phases Flow in Heterogeneous reservoirs: Current trends. Oil \& Gas Science and Technology. Rev. IFP 59 (2004) 185-195.

[4] D. Bailly, R. Ababou, M. Quintard. Characterization and hydraulic behaviour of hoghly fissured geologic media (Karsts). Proc. internat. Symp. IAHR-GW (Flow \& transport in heterogeneous subsurface formations : theory, modelling \& applications), Istanbul, Turkey,2008.

[5] J.P. Chilès, P. Delfiner. Geostatistics - modelling spatial uncertainty, Wiley\&Sons, New York, 1999.

[6] S.E. Gasda, M.A. Celia. Upscaling relative permeabilities in a structured porous medium. Adv. Water Res. 28 (2005) 493-506.

[7] C. He, L.J. Durlofsky. Structured flow-baes gridding and upscaling for modelling subsurface flow. Adv. Water Resour. Res, 29 (2006) 1876-1892.

[8] D. T. Hristopulos. Renormalization group methods in subsurface hydrology: overview and applications in hydraulic conductivity upscaling. Adv. Water Res. 26 (2003) 1279-1308.

[9] P. Jenny, S. H. Lee, H. A. Tchelepi. Multi-scale finite volume method for elliptic problems in subsurface flow simulation. Journal of Computational Physics, 187 (2003) 47-67

[10] M. Kfoury. Changement d'échelle séquentiel pour des milieux fracturés hétérogènes. Thèse de doctorat, INPT, Institut de mécanique des fluides de Toulouse. 2004.

[11] M. Kfoury, R. Ababou, B. Noetinger, M. Quintard. Upscaling fractured heterogeneous media : permeability and mass exchange coefficient. Journal of applied mechanics, transactions ASME, $73(2006) 41-46$

[12] M.J. King. Recent Advances in Upgridding. Oil \& Gas Science and Technology. Rev. IFP 62 (2007) 195-205.

[13] V. Lagneau. R2D2 - Reactive Transport and Waterflow on an Odd Dimension 2 grid, Rapport Technique, Ecole des mines de Paris, 2007.

[14] J. van der Lee, L. De Windt, V. Lagneau, P. Goblet. Presentation and application of the reactive transport code Hytec. Computational methods in water resources 1 (2002) 599-606.

[15] G. de Marsily. Hydrologie quantitative, Ed. Masson Paris (1981)

[16] G. Matheron. Eléments pour une théorie des milieux poreux. Masson. Paris. 1967.

[17] A. Pouya. Tenseurs de perméabilité équivalente d'un domaine hétérogène fini. C.R. Geoscience 337 (2005) 581-588.

[18] P. Renard. Modélisation des écoulements en milieux poreux hétérogènes. Calcul des perméabilités équivalentes. Mémoire des sciences de la Terre n. 32. Ecole des mines de Paris. 1997.

[19] P. Renard, G. de Marsily. Calculating equivalent permeability: a review. Adv. Water Resour. Res, 20 (1997) 253-278.

[20] P. Renard, G. Le Loc'h, E. Ledoux, G. de Marsily, R. Mackay. A fast algorithm for the estimation of the equivalent hydraulic conductivity of heterogeneous porous media. Water Resouces Research, 36(12)3567-3580, 2000.

[21] F. Ubertosi, F. Delay, J. Bodin, G. Porel. A new method for generating a pipe network to handle channelled flow in fractured rocks. C. R. Geoscience 339 (2007) 682-691 